\title{
Associations between amino acids from vegetable and animal sources and body composition in a female cohort
}

\author{
A. Jennings ${ }^{1}$, A. Cassidy ${ }^{1}$, T. Spector ${ }^{2}$, A. Macgregor ${ }^{1}$ and A. Welch ${ }^{1}$ \\ ${ }^{1}$ Department of Nutrition, Norwich Medical School, University of East Anglia, Norwich, NR4 7TJ and \\ ${ }^{2}$ Department of Twin Research and Genetic Epidemiology, King's College, London SE1 7EH, UK
}

Protein and the branched chain amino acids (BCAA) leucine, isoleucine and valine have been related to a lower prevalence of obesity ${ }^{(1)}$, however, a review examining the effect of protein from animal and vegetable sources on body composition highlighted a paucity of studies assessing habitual protein intakes and precisely measured body composition ${ }^{(2)}$.

Cross-sectional data from 2854 females in the Twins UK cohort was analysed to examine associations between intake of total protein and BCAA from animal and vegetable sources, estimated from food frequency questionnaires, and objectively measured body composition. Differences in BMI, total fat and central body fat, measured by dual-energy X-ray absorptiometry, were examined between extreme quintiles of intake.

The mean age of participants was 48.2 years (IQR 40-58), BMI $25.1 \mathrm{~kg} / \mathrm{m}^{2}$ (IQR 22-27), total body fat 34.7\% (IQR 30-40) and central body fat $30.1 \%$ (IQR 22-38). Protein intake was $80.7 \mathrm{~g}$ /day (IQR 66-94) with vegetable protein accounting for $38.5 \%$ of intake. BCAA intake was $14.6 \mathrm{~g}$ /day (IQR 12-17) and contributed to $18.1 \%$ of total protein (6.4\% vegetable sources).

In multivariate models, including age, physical activity, smoking, dietary under-reporting and intakes of energy (kcal/d), fibre ( $\mathrm{g} / \mathrm{d})$, carbohydrate (\% energy), fat (\% energy) and protein ( $\%$ energy), participants with a higher proportion of protein from vegetable sources had lower BMI and body fat with the converse found for participants with a higher proportion of protein from animal sources (all $P<0.01)$. Similarly, participants with a higher proportion of BCAA from vegetable sources had a healthier body composition profile with the converse found for participants with a higher proportion from animal sources (all $P<0.01$ ).

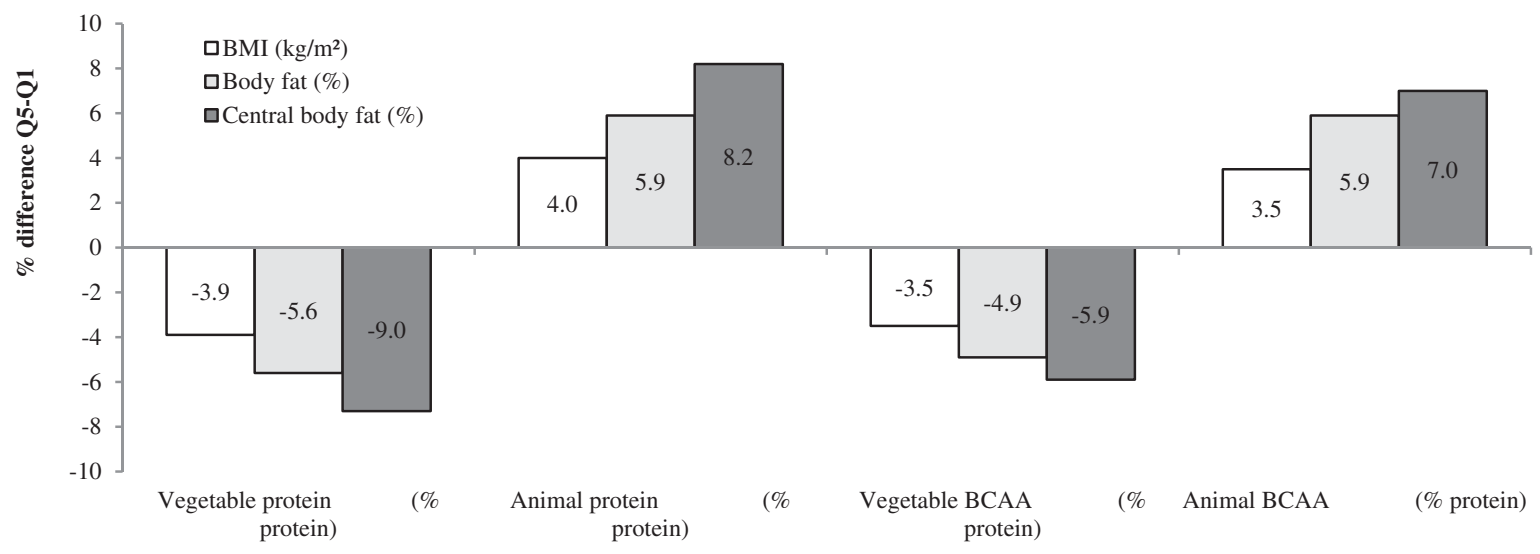

Fig. 1. Differences in weight status between extreme quintiles of amino acid intake.

In conclusion, a higher proportion of protein and BCAA from vegetable sources was associated with lower BMI and percentage body fat in females. The greatest associations were found for central body fat which is a major contributor to the development of insulin resistance, hypertension and the metabolic syndrome ${ }^{(3)}$. The underlying reason for the direction of the association between body composition and intakes of protein and BCAA from animal sources merits further investigation.

1. Quin L, Xun P \& Bujnowski D (2011) J Nutr 141, 249-254.

2. Gilbert JA, Bendssen NT, Tremblay A et al. (2011) Nutr Metab Cardiovasc Dis 21, B16-31.

3. Kopelman P (2007) Obes Rev 8, 13-17. 